\title{
Endovascular Therapy for Acute Limb Ischemia
}

\author{
Makoto Utsunomiya
}

Division of Cardiovascular Medicine, Toho University Ohashi Medical Center, Tokyo, Japan

\section{See article vol. 28: 000-000}

A surgical approach, including surgical thrombectomy, would be the first choice for revascularization in patients with acute limb ischemia (ALI). ALI is a serious limb- and life-threatening disease with extremely poor prognosis.

Although various factors are considered as the cause of ALI, it is basically caused by an acute occlusion of the vessel by a thrombus. An occlusion present in the proximal region leads to a worse prognosis. The amount of thrombi increases when the occlusion occurs in the proximal region, and because effective treatment device for thrombotic lesion has not yet been approved in Japan, there are some difficulties in treating lesions with a lot of thrombi by endovascular therapy (EVT).

Surgical thrombectomy, however, is not always the easy applicable treatment because many patients with ALI are old and sometimes are bedridden compared with patients with chronic symptomatic peripheral arterial disease. Surgical thrombectomy is also not applicable when certain amount of time has passed from ALI onset because myonephropathic metabolic syndrome risks should be considered. It is difficult to treat such patients by surgical procedure or EVT; therefore, primary amputation or observation/ conservative management is selected in most cases.

In recent years, a novel transcatheter treatment device for thrombotic lesions has been developed in Western countries. Because the device shows favorable results, the number of patients with ALI who were treated by EVT has been increasing where the surgical procedure is not applicable. In recently reported research using the US national database, the number of surgical revascularization and EVT are applied to patients with ALI is almost the same. When comparing surgical procedure with EVT by propensity score matching, the number of in-hospital deaths is significantly lower in EVT group and no differences has been shown in the amputation rate between groups ${ }^{1)}$. Although promising results are shown in the report, EVT cannot immediately be the first choice for ALI treatment in Japan. Addressing the concern of device lag in EVT field to facilitate effective EVT in patients with ALI who are not suitable for surgical procedure is desirable.

This study ${ }^{2)}$ reviews the results of EVT in patients with ALI using large-scale research in Japan. Because the rate of patients with ALI in all EVT cases is only approximately $3 \%$, it is imaginable that many operators are not given any opportunities to perform EVT in a patient with ALI in one year. It is very significant to review the results of EVT on such serious and rare disease and its treatment from a largescale database. Unfortunately, suitable conditions for EVT remain unknown because in this study, it is unclear which patients received EVT and which patients received surgical revascularization. However, it is clinically significant that the risk of in-hospital complications was verified from this study. Because long-term results are not available and the true implication of this study cannot be concluded, we believe that this study has great significance in knowing the background and clinical presentations of patients with ALI.

The development of device, the improvement of clinical results, and the comparative study of EVT with surgical revascularization are expected in the near future.

\section{Conflicts of Interest}

None.

\section{References}

1) Kolte D, Kennedy KF, Shishehbor MH, Mamdani ST, Stangenberg L, Hyder ON, Soukas P, Aronow HD.

Address for correspondence: Makoto Utsunomiya, Division of Cardiovascular Medicine, Toho University Ohashi Medical Center, 2-22-36 Ohashi, Meguro-ku, Tokyo, Japan E-mail:m.utsu0705@gmail.com

Received: December 16, 2020 Accepted for publication: December 25, 2020

Copyright@2021 Japan Atherosclerosis Society

This article is distributed under the terms of the latest version of CC BY-NC-SA defined by the Creative Commons Attribution License.

\section{Advance Publication Journal of Atherosclerosis and Thrombosis}


Endovascular Versus Surgical Revascularization for Acute Limb Ischemia. A Propensity-Score Matched Analysis. Circ Cardiovasc Interv, 2020; 13: e008150. DOI: 10.1161/CIRCINTERVENTIONS.119.008150

2) Tsujimura T, Takahara M, Iida $O$, Kohsaka S, Soga $Y$, Fujihara M, Mano T, Ohya M, Shinke T, Amano T, Ikari $\mathrm{Y}$, on behalf of the J-EVT and J-PCI investigators, The
Japanese Association of Cardiovascular Intervention and Therapeutics. In-Hospital Outcomes after Endovascular Therapy for Acute Limb Ischemia: A Report from a Japanese Nationwide Registry [J-EVT Registry]. J Atheroscler Thromb, 2020; in press. DOI: http://doi. org/10.5551/jat.60053 